\title{
Fórmula Explícita e Interpretação Combinatória para os Números de Fibonacci ${ }^{1}$
}

I.M. CRAVEIRO², UFMS-DMT, Cidade Universitária, Cx.P. 549, 79070-900 Campo Grande, MS, Brasil

J.P.O. SANTOS ${ }^{3}$, Instituto de Matemática, Estatística e Ciências Computacional, DMA, UNICAMP, Cx.P. 6065, 13081-970 Campinas, SP, Brasil.

Resumo. Neste trabalho, damos uma nova interpretação combinatória para os números de Fibonacci em termos de partições restritas, fazendo uso do Símbolo de Frobenius. Também damos uma demonstração de uma conjectura para uma fórmula explícita de uma família de polinômios dada por Santos em [9].

\section{Introdução}

Santos, em [9], seguindo um método introduzido por Andrews em [1], define funções de duas variáveis $f(q, t)$ associadas a identidades do tipo Rogers-Ramanujan. Consideramos, inicialmente, as funções de duas variáveis associadas às identidades (20) e (16) de Slater que são, respectivamente

$$
\begin{aligned}
& f_{20}(q, t)=\sum_{n=0}^{\infty} \frac{t^{n} q^{n^{2}}}{\left(t ; q^{2}\right)_{n+1}\left(-t q^{2} ; q^{2}\right)_{n}} \\
& f_{16}(q, t)=\sum_{n=0}^{\infty} \frac{t^{n} q^{n^{2}+2 n}}{\left(t ; q^{2}\right)_{n+1}\left(-t q^{2} ; q^{2}\right)_{n}},
\end{aligned}
$$

onde $(a ; q)_{n}=(1-a)(1-a q) \ldots\left(1-a q^{n-1}\right)$ e $n$ é um inteiro não negativo.

Inserindo-se um parâmetro $k$ na função de duas váriaveis $f_{16}(q, t)$, definimos a família de funções

$$
f_{k}(q, t)=\sum_{n=0}^{\infty} \frac{t^{n} q^{n^{2}+2 k n}}{\left(t ; q^{2}\right)_{n+1}\left(-t q^{2} ; q^{2}\right)_{n}} .
$$

Através de simples manipulações em (1.1), obtemos a seguinte equação funcional para $f_{k}(q, t)$

$$
(1-t)\left(1+t q^{2}\right) f_{k}(q, t)=1+t q^{2}+t q^{2 k+1} f_{k}\left(q, t q^{2}\right)
$$

\footnotetext{
${ }^{1}$ Este trabalho recebeu auxílio do $\mathrm{CNPq}$

2irene@dmt.ufms.br

3 josepli@ime.unicamp.br
} 
e, substituindo $f_{k}(q, t)=\sum_{n=0}^{\infty} P_{n}^{k}(q) t^{n}$ em $(1.2)$, temos que

$$
\left\{\begin{array}{l}
P_{0}^{k}(q)=1 \\
P_{1}^{k}(q)=1+q^{2 k+1} \\
P_{n}^{k}(q)=\left(1-q^{2}+q^{2 n-1+2 k}\right) P_{n-1}^{k}(q)+q^{2} P_{n-2}^{k}(q)
\end{array}\right.
$$

Chamamos de coeficientes trinomiais aos coeficientes de $x^{j}$ na expansão de

$$
\left(1+x+x^{2}\right)^{n}
$$

que são dados por $\left(\begin{array}{c}n \\ j\end{array}\right)_{2}=\sum_{h=0}^{m}(-1)^{h}\left(\begin{array}{l}n \\ h\end{array}\right)\left(\begin{array}{l}2 n-2 h \\ n-j-h\end{array}\right)$.

Fazemos uso das seguintes famílias de polinômios que são $q$-análogos dos coeficientes trinomiais no mesmo sentido em que os polinômios de Gauss $\left[\begin{array}{l}n \\ j\end{array}\right]$ são qanálogos dos coeficientes binomiais,

$$
\begin{aligned}
& T_{0}(m, A)=T_{0}(m, A, q)=\sum_{j=0}^{m}(-1)^{j}\left[\begin{array}{c}
m \\
j
\end{array}\right]_{q^{2}}\left[\begin{array}{c}
2 m-2 j \\
m-A-j
\end{array}\right], \\
& T_{1}(m, A)=T_{1}(m, A, q)=\sum_{j=0}^{m}(-q)^{j}\left[\begin{array}{c}
m \\
j
\end{array}\right]_{q^{2}}\left[\begin{array}{c}
2 m-2 j \\
m-A-j
\end{array}\right] .
\end{aligned}
$$

Definindo $U(m, A)=U(m, A, q)=T_{0}(m, A)+T_{0}(m, A+1)$, temos em [2] as seguintes relações que serão fundamentais em nossas demonstrações

$$
\begin{gathered}
U(m, A)=\left(1+q^{2 m-1}\right) U(m-1, A)+q^{m-A} T_{1}(m-1, A-1) \\
+q^{m+A+1} T_{1}(m-1, A+2), \\
T_{0}(m, A)=T_{0}(m-1, A-1)+q^{m+A} T_{1}(m-1, A)+q^{2 m+2 A} T_{0}(m-1, A+1), \\
T_{1}(m, A)=T_{1}(m-1, A)+q^{m+A} T_{0}(m-1, A+1)+q^{m-A} T_{0}(m-1, A-1), \\
T_{1}(m, A)-q^{m-A} T_{0}(m, A)-T_{1}(m, A+1)+q^{m+A+1} T_{0}(m, A+1)=0 .
\end{gathered}
$$

\section{Uma Fórmula Explícita para os Números de Fibonacci}

Santos em [8], provou uma fórmula explícita para a família de polinômios $P_{n}^{k}$ para o caso $k=0$. Para o caso $k=1$, temos a seguinte conjecturada dada em [9]:

$$
c(n)=\sum_{j=-\infty}^{\infty} q^{10 j^{2}+3 j} U(n, 5 j)-\sum_{j=-\infty}^{\infty} q^{10 j^{2}+13 j+4} U(n, 5 j+3),
$$

onde $c(n)=P_{n-1}^{1}$. 
Uma nova fórmula para os números de Fibonacci em função dos coeficientes trinomiais é obtida uma vez que, para $q=1,(1.3)$ nos fornece a relação de recorrência que define os números de Fibonacci

$$
\lim _{q \rightarrow 1} c(n)=F_{n-1}=\sum_{\mathrm{J}=-\infty}^{\infty}\left\{\left(\begin{array}{c}
n+1 \\
5 j+1
\end{array}\right)_{2}-\left(\begin{array}{c}
n+1 \\
5 j+3
\end{array}\right)_{2}\right\},
$$

onde $P_{n-1}^{1}(1)=F_{n-1}$ e $F_{n}$ denota o número de Fibonacci de posição $n$.

Teorema 2.1. A fórmula c(n) dada em (2.1) satisfaz à relação de recorrência e às mesmas condiçôes iniciais dadas em (1.3) quando fazemos $k=1$.

Demonstração:

Sejam $A$ e $n$ inteiros positivos. Consideremos a seguinte expressão

$$
U(n+1, A)-\left(1-q^{2}+q^{2 n+1}\right) U(n, A)-q^{2} U(n-1, A) .
$$

Substituindo em (2.2) a definição de $U(m, A)$ e fazendo uso da identidade dada em (1.4), temos que

$$
\begin{aligned}
& U(n+1, A)-\left(1+q^{2 n+1}\right) U(n, A)+q^{2} U(n, A)-q^{2} U(n-1, A) \\
& =q^{n+1-A} T_{1}(n, A-1)+q^{n+A+2} T_{1}(n, A+2)+q^{2} T_{0}(n, A) \\
& \quad+q^{2} T_{0}(n, A+1)-q^{2} T_{0}(n-1, A)-q^{2} T_{0}(n-1, A+1) .
\end{aligned}
$$

Observamos que $T_{0}(m,-A)=T_{0}(m, A)$ e $T_{1}(m, A)=T_{1}(m,-A)$. Substituindo (1.5) e (1.6), após alguns cancelamentos, obtemos que o segundo membro de (2.3) é igual a

$$
\begin{aligned}
& q^{n+1-A} T_{1}(n-1, A-1)+q^{2 n} T_{0}(n-1, A)+q^{2 n-2 A+2} T_{0}(n-1, A-2) \\
& +q^{n+A+2} T_{1}(n-1, A+2)+q^{2 n+2 A+4} T_{0}(n-1, A+3) \\
& +q^{2 n} T_{0}(n-1, A+1)+q^{n-A+2} T_{1}(n-1, A)+q^{2 n-2 A+2} T_{0}(n-1, A-1) \\
& +q^{n+A+3} T_{1}(n-1, A+1)+q^{2 n+2 A+4} T_{0}(n-1, A+2) .
\end{aligned}
$$

Observamos que se verificarmos que a expressão (2.4), quando substituída correspondentemente em $U(n, 5 j)$ e em $U(n, 5 j+3)$ que aparecem em $(2.1)$, nos conduzir a uma expressão identicamente nula, estaremos provando que $c(n)$ satisfaz (1.3). Assim, fazendo essas duas substituções em (2.1) e após alguns cancelamentos, obtemos 


$$
\begin{aligned}
& \sum_{j=-\infty}^{\infty} q^{n+10 j^{2}-2 j+1} T_{0}(n-1,5 j-1)+\sum_{j=-\infty}^{\infty} q^{2 n+10 j^{2}+3 j} T_{0}(n-1,5 j) \\
& +\sum_{j=-\infty}^{\infty} q^{2 n+10 j^{2}-7 j+2} T_{0}(n-1,5 j-2)+\sum_{j=-\infty}^{\infty} q^{n+10 j^{2}-2 j+2} T_{1}(n-1,5 j) \\
& +\sum_{j=-\infty}^{\infty} q^{2 n+10 j^{2}-7 j+2} T_{0}(n-1,5 j-1)+\sum_{j=-\infty}^{\infty} q^{n+10 j^{2}+8 j+3} T_{1}(n-1,5 j+1) \\
& +\sum_{j=-\infty}^{\infty} q^{2 n+10 j^{2}+13 j+4} T_{0}(n-1,5 j+2)-\sum_{j=-\infty}^{\infty} q^{n+10 j^{2}+18 j+9} T_{1}(n-1,5 j+5) \\
& -\sum_{j=-\infty}^{\infty} q^{2 n+10 j^{2}+23 j+14} T_{0}(n-1,5 j+6)-\sum_{j=-\infty}^{\infty} q^{2 n+10 j^{2}+13 j+4} T_{0}(n-1,5 j+4) \\
& -\sum_{j=-\infty}^{\infty} q^{n+10 j^{2}+8 j+3} T_{1}(n-1,5 j+3)-\sum_{j=-\infty}^{\infty} q^{2 n+10 j^{2}+3 j} T_{0}(n-1,5 j+2) \\
& -\sum_{j=-\infty}^{\infty} q^{n+10 j^{2}+18 j+10} T_{1}(n-1,5 j+4)-\sum_{j=-\infty}^{\infty} q^{2 n+10 j^{2}+23 j+14} T_{0}(n-1,5 j+5) .
\end{aligned}
$$

Considerando a quarta e décima terceira parcelas e fazendo a substituição $j \rightarrow j+1$ na quarta parcela, segue de (1.7) que

$$
\begin{gathered}
\sum_{j=-\infty}^{\infty} q^{n+10 j^{2}-2 j+2} T_{1}(n-1,5 j)-\sum_{j=-\infty}^{\infty} q^{n+10 j^{2}+18 j+10} T_{1}(n-1,5 j+4) \\
=\sum_{j=-\infty}^{\infty} q^{n+10 j^{2}+18 j+10}\left(T_{1}(n-1,5 j+5)-T_{1}(n-1,5 j+4)\right) \\
=-\sum_{j=-\infty}^{\infty} q^{2 n+10 j^{2}+13 j+5} T_{0}(n-1,5 j+4) \\
+\sum_{j=-\infty}^{\infty} q^{2 n+10 j^{2}+23 j+14} T_{0}(n-1,5 j+5) .
\end{gathered}
$$

Substituindo (2.6) em (2.5) temos que a segunda soma dada em (2.6) cancela-se com a décima quarta dada em (2.5). E a primeira soma dada em (2.6) cancela-se com a quinta dada em (2.5) após fazermos a substituição $j \rightarrow j+1$. Assim vemos 
que (2.5) é igual a

$$
\begin{aligned}
& \sum_{j=-\infty}^{\infty} q^{n+10 j^{2}-2 j+1} T_{1}(n-1,5 j-1)+\sum_{j=-\infty}^{\infty} q^{2 n+10 j^{2}+3 j} T_{0}(n-1,5 j) \\
& +\sum_{j=-\infty}^{\infty} q^{2 n+10 j^{2}-7 j+2} T_{0}(n-1,5 j-2)+\sum_{j=-\infty}^{\infty} q^{n+10 j^{2}+8 j+3} T_{1}(n-1,5 j+1) \\
& +\sum_{j=-\infty}^{\infty} q^{2 n+10 j^{2}+13 j+4} T_{0}(n-1,5 j+2)-\sum_{j=-\infty}^{\infty} q^{n+10 j^{2}+18 j+9} T_{1}(n-1,5 j+5) \\
& -\sum_{j=-\infty}^{\infty} q^{2 n+10 j^{2}+23 j+14} T_{0}(n-1,5 j+6)-\sum_{j=-\infty}^{\infty} q^{2 n+10 j^{2}+13 j+4} T_{0}(n-1,5 j+4) \\
& -\sum_{j=-\infty}^{\infty} q^{n+10 j^{2}+8 j+3} T_{1}(n-1,5 j+3)-\sum_{j=-\infty}^{\infty} q^{2 n+10 j^{2}+3 j} T_{0}(n-1,5 j+2) .
\end{aligned}
$$

Considerendo a primeira e sexta parcelas, fazendo a substituição $j \rightarrow j+1$ na primeira parcela, segue de (1.7),

$$
\begin{aligned}
& \sum_{j=-\infty}^{\infty} q^{n+10 j^{2}-2 j+1} T_{1}(n-1,5 j-1)-\sum_{j=-\infty}^{\infty} q^{n+10 j^{2}+18 j+9} T_{1}(n-1,5 j+5) \\
= & \sum_{j=-\infty}^{\infty} q^{n+10 j^{2}+18 j+10}\left(T_{1}(n-1,5 j+4)-T_{1}(n-1,5 j+5)\right) \\
= & \sum_{j=-\infty}^{\infty} q^{2 n+10 j^{2}+13 j+4} T_{0}(n-1,5 j+4)-\sum_{j=-\infty}^{\infty} q^{2 n+10 j^{2}+23 j+13} T_{0}(n-1,5 j+5) .
\end{aligned}
$$

Substituindo (2.8) em (2.7), temos que a segunda parcela (fazendo $j \rightarrow j+1$ ) e a oitava parcela se cancelam. Reescrevendo

$$
\begin{aligned}
& \sum_{j=-\infty}^{\infty} q^{2 n+10 j^{2}-7 j+2} T_{0}(n-1,5 j-2)+\sum_{j=-\infty}^{\infty} q^{n+10 j^{2}+8 j+3} T_{1}(n-1,5 j+1) \\
& +\sum_{j=-\infty}^{\infty} q^{2 n+10 j^{2}+13 j+4} T_{0}(n-1,5 j+2)-\sum_{j=-\infty}^{\infty} q^{2 n+10 j^{2}+23 j+14} T_{0}(n-1,5 j+6) \\
& -\sum_{j=-\infty}^{\infty} q^{n+10 j^{2}+8 j+3} T_{1}(n-1,5 j+3)-\sum_{j=-\infty}^{\infty} q^{2 n+10 j^{2}+3 j} T_{0}(n-1,5 j+2) .
\end{aligned}
$$


Fazendo a substituição $j \rightarrow j-1$ na quarta parcela e $j \rightarrow j+1$ na primeira parcela,

$$
\begin{aligned}
& \sum_{j=-\infty}^{\infty} q^{2 n+10 j^{2}+13 j+5} T_{0}(n-1,5 j+3)+\sum_{j=-\infty}^{\infty} q^{n+10 j^{2}+8 j+3} T_{1}(n-1,5 j+1) \\
& +\sum_{j=-\infty}^{\infty} q^{2 n+10 j^{2}+13 j+4} T_{0}(n-1,5 j+2)-\sum_{j=-\infty}^{\infty} q^{2 n+10 j^{2}+3 j+1} T_{0}(n-1,5 j+1) \\
& -\sum_{j=-\infty}^{\infty} q^{n+10 j^{2}+8 j+3} T_{1}(n-1,5 j+3)-\sum_{j=-\infty}^{\infty} q^{2 n+10 j^{2}+3 j} T_{0}(n-1,5 j+2) .
\end{aligned}
$$

Segue de (1.7)

$$
\begin{aligned}
& -\sum_{j=-\infty}^{\infty} q^{n+10 j^{2}+8 j+3} T_{1}(n-1,5 j+3)=-\sum_{j=-\infty}^{\infty} q^{n+10 j^{2}+8 j+3} T_{1}(n-1,5 j+2) \\
& +\sum_{j=-\infty}^{\infty} q^{2 n+10 j^{2}+3 j} T_{0}(n-1,5 j+2)-\sum_{j=-\infty}^{\infty} q^{2 n+10 j^{2}+13 j+5} T_{0}(n-1,5 j+3) .
\end{aligned}
$$

Substituindo (2.10) em (2.9), obtemos

$$
\begin{aligned}
& \sum_{j=-\infty}^{\infty} q^{2 n+10 j^{2}+8 j+3} T_{1}(n-1,5 j+1)+\sum_{j=-\infty}^{\infty} q^{2 n+10 j^{2}+13 j+4} T_{0}(n-1,5 j+2) \\
& -\sum_{j=-\infty}^{\infty} q^{2 n+10 j^{2}+3 j+1} T_{0}(n-1,5 j+1)-\sum_{j=-\infty}^{\infty} q^{n+10 j^{2}+8 j+3} T_{1}(n-1,5 j+2) .
\end{aligned}
$$

Considerando a primeira e a quarta parcelas, segue de (1.7)

$$
\begin{aligned}
& -\sum_{j=-\infty}^{\infty} q^{n+10 j^{2}+8 j+3} T_{1}(n-1,5 j+1)-\sum_{j=-\infty}^{\infty} q^{n+10 j^{2}+8 j+3} T_{1}(n-1,5 j+2) \\
& =-\sum_{j=-\infty}^{\infty} q^{n+10 j^{2}+8 j+3}\left(T_{1}(n-1,5 j+1)-T_{1}(n-1,5 j+2)\right) \\
& =\sum_{j=-\infty}^{\infty} q^{2 n+10 j^{2}+3 j+1} T_{0}(n-1,5 j+1)-\sum_{j=-\infty}^{\infty} q^{2 n+10 j^{2}+13 j+4} T_{0}(n-1,5 j+2) .
\end{aligned}
$$

Portanto

$$
\begin{aligned}
& \sum_{j=-\infty}^{\infty} q^{2 n+10 j^{2}+8 j+3} T_{1}(n-1,5 j+1)+\sum_{j=-\infty}^{\infty} q^{2 n+10 j^{2}+13 j+4} T_{0}(n-1,5 j+2) \\
& -\sum_{j=-\infty}^{\infty} q^{2 n+10 j^{2}+3 j+1} T_{0}(n-1,5 j+1)-\sum_{j=-\infty}^{\infty} q^{n+10 j^{2}+8 j+3} T_{1}(n-1,5 j+2)=0
\end{aligned}
$$

o que conclui a demonstração. 


\section{Uma Interpretação Combinátoria para os Números de Fibonacci}

Consideremos o gráfico de Ferrers da partição $4+3+1+1$

$$
\vdots: \cdot \quad \leftrightarrow\left(\begin{array}{ll}
3 & 1 \\
3 & 0
\end{array}\right)
$$

o símbolo de Frobenius para essa partição é dado pela matriz em (3.1). Dada uma partição $\pi$, o símbolo de Frobenius de $\pi$ é dado da seguinte forma:

$$
\left(\begin{array}{llll}
a_{11} & a_{12} & \ldots & a_{1 n} \\
a_{21} & a_{22} & \ldots & a_{2 n}
\end{array}\right)
$$

onde $n$ é a dimensão do quadrado de Durfee, $a_{1 j}$ é o número de pontos que estão à direita da diagonal deste quadrado e na $j$-ésima linha deste gráfico e $a_{2 j}$ é o número de pontos que estão abaixo da diagonal deste quadrado e na $j$-ésima coluna deste gráfico (lendo da esquerda para direita). O elemento $a_{1 n}$ dado em (3.2) é chamado elemento do topo.

Definição 3.1. Dizemos que uma partição é Frobenius de alternância par (ímpar) se os elementos da primeira linha da matriz dada em (3.2) alternam a paridade, sendo que $a_{1 n}$ é par (ímpar).

Nesta seção vamos provar que o coeficiente de $t^{N}$ na expansão de $f_{k}(q, t)$ dada por (1.1), que é $P_{N}^{k}(q)$ dado em (1.3) é a função geradora para partições Frobenius de alternância ímpar (par) se $k$ é ímpar (par), com maior parte menor do que ou igual a $N+k$ e o elemento do topo $\geq k$.

Podemos escrever (1.1) da seguinte forma:

$$
\begin{aligned}
f_{k}(q, t) & =\sum_{n=0}^{\infty} \frac{t^{n} q^{n^{2}+2 n k}}{(1-t)\left(t^{2} q^{4} ; q^{4}\right)_{n}} \\
& =\frac{1}{1-t} \sum_{n=0}^{\infty} \frac{t^{n} q^{n^{2}+2 n k}}{\left(1-t^{2} q^{4}\right)\left(1-t^{2} q^{8}\right) \ldots\left(1-t^{2} q^{4 n}\right)} .
\end{aligned}
$$

Em

$$
\left(t^{2} q^{4} ; q^{4}\right)_{n}=\left(1-t^{2} q^{4}\right)\left(1-t^{2} q^{8}\right) \ldots\left(1-t^{2} q^{4 j}\right) \ldots\left(1-t^{2} q^{4 n}\right),
$$

vamos enumerar os fatores como primeiro fator, segundo fator, $\ldots, n$-ésimo fator (lendo da esquerda para à direita).

É fácil ver que o expoente de $q$ (dado em (3.4)) é um múltiplo de 4 e também de $j$, onde $j$ é a posição do fator no produto em (3.4). Dessa forma, se dividirmos o expoente de $q$ por 2 o resultado é sempre um número par e múltiplo de $j$. Desta observação trivial temos que na expansão de $\frac{1}{\left(1-t^{2} q^{4 j}\right)}$, que é

$$
1+\left(t^{2} q^{4 j}\right)^{1}+\left(t^{2} q^{4 j}\right)^{2}+\ldots+\left(t^{2} q^{4 j}\right)^{i}+\ldots,
$$


o expoente de $q$ quando dividido por $2 j$ é sempre igual ao expoente de $t$ que também é par. Agora podemos explicar como construir partições Frobenius de alternância ímpar (par) se $k$ é ímpar (par), com maior parte menor do que ou igual a $N+k$ e o elemento do topo $\geq k$, do coeficiente de $t^{N}$ em $f_{k}(q, t)$. O seguinte exemplo deixará claro como é estabelecido o procedimento que gera partições deste tipo. Seja $\frac{t^{n} q^{n^{2}+2 n k}}{\left(t^{2} q^{4} ; q^{4}\right)_{n}}$ para $n=3$ e $k=1$.

$$
\begin{aligned}
\frac{t^{3} q^{3^{2}+2.3 .1}}{\left(t^{2} q^{4} ; q^{4}\right)_{3}}= & \frac{t^{3} q^{3^{2}+2.3 .1}}{\left(1-t^{2} q^{4}\right)\left(1-t^{2} q^{8}\right)\left(1-t^{2} q^{12}\right)} \\
= & t^{3} q^{3^{2}+2.3 .1}\left(1+t^{2} q^{4}+t^{4} q^{8} \ldots\right) \\
& \left(1+t^{2} q^{8}+t^{4} q^{16}+\ldots\right)\left(1+t^{2} q^{12}+t^{4} q^{24}+\ldots\right) .
\end{aligned}
$$

Associamos $t^{3} q^{3^{2}}$ ao quadrado de Durfee $3 \times 3$, o expoente de $t$ contribue para a maior parte desta partição que estamos construindo. Começamos com um quadrado de Durfee $3 \times 3$

Abaixo e ao lado do quadrado de Durfee acrescentamos um retângulo de altura 3 e base 1, estes retângulos são associados ao expoente de $q^{2.3 .1}$,

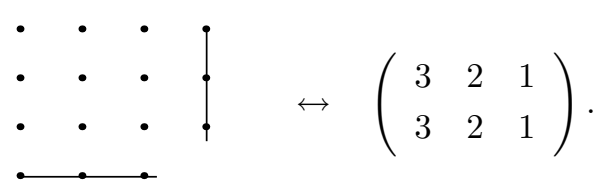

Consideremos o termo $t^{2} q^{12}$ do terceiro fator dado em (3.6); dividimos o expoente de $q$ por 2, obtendo 6 como resultado; depois dividimos 6 por 3 (posição do fator), cujo o resultado é 2. Agora colocamos os pontos à direita do retângulo de base 1 e altura 3 como uma pilha de altura 3 e largura 2. Acrescentamos a mesma pilha abaixo do retângulo de altura $k$ e base $n$,

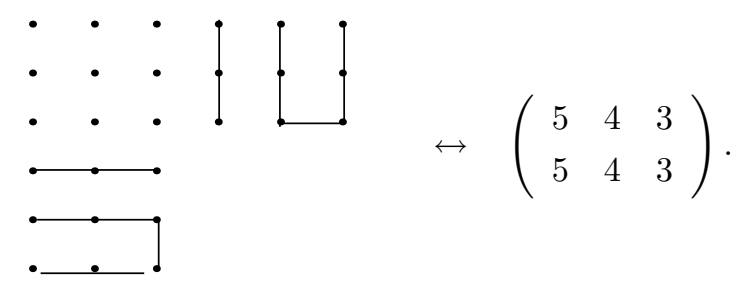

Com o termo $t^{2} q^{8}$ do segundo fator: dividimos o expoente de $q$ por 2 e obtemos 4 ; depois dividimos o resultado por 2 (posição do fator), obtendo 2. Colocamos estes 
quatro pontos como uma pilha de largura 2 (expoente de $t$ ) e altura 3 (a posição do fator) ao lado e abaixo do retângulo de base 2 e altura 3 ,

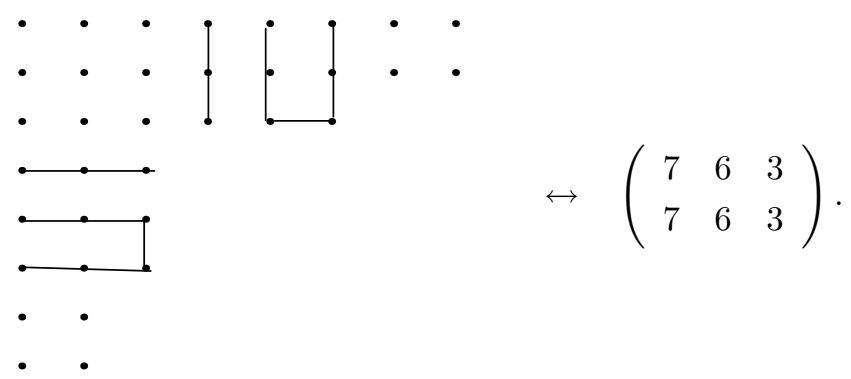

Para o termo $t^{4} q^{8}$ do primeiro fator, dividimos o expoente de $q$, por 2 . O resultado é dividido por 1 (posição do fator). Colocamos estes quatro pontos em uma pilha de largura 4 (expoente de $t$ ) e altura 1 (posição do fator) ao lado e abaixo do retângulo de base 2 e altura 2 ,

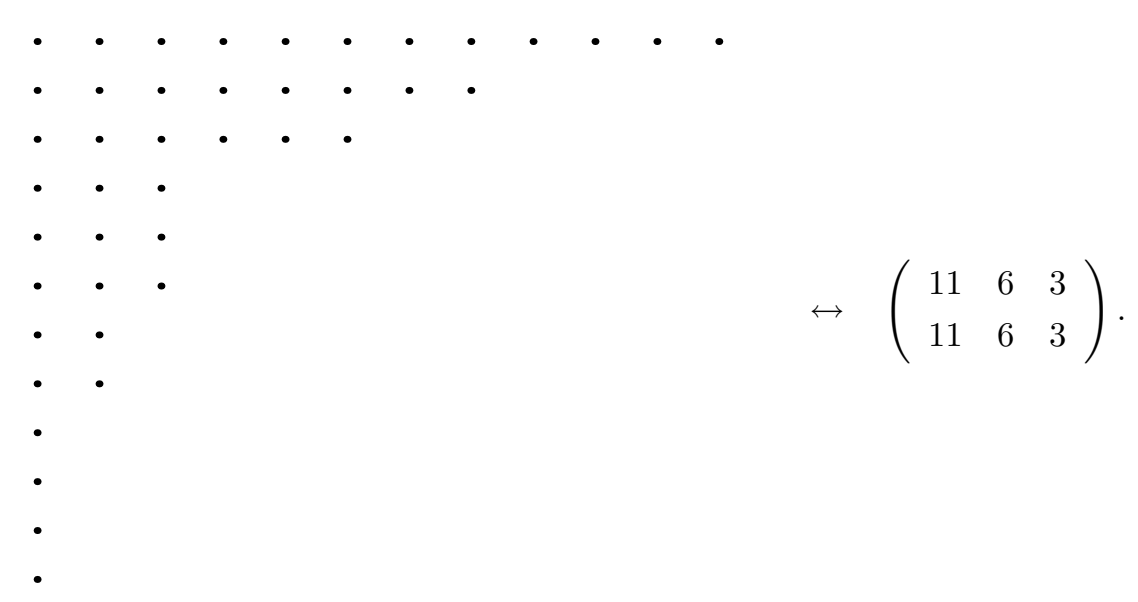

Observamos que estes 43 pontos são gerados por

$$
t^{3} q^{15}\left(t^{2} q^{12}\right)\left(t^{2} q^{8}\right)\left(t^{4} q^{8}\right)=t^{11} q^{43} .
$$

Com este procedimento, representamos uma partição do inteiro 43 autoconjugada Frobenius de alternância ímpar (par), caso $k$ seja ímpar (par), com maior parte igual a 12. Considerando o fator $\frac{1}{1-t}$, podemos concluir que $P_{N}^{k}$, o coeficiente de $t^{N}$ em $f_{k}(q, t)$, é a função geradora para partições autoconjugadas Frobenius de alternância ímpar (par, caso $k$ seja par) com maior parte menor do que ou igual a $N+k$ e o elemento do topo $\geq k$. Para mostrar que o processo é reversível procedemos da seguinte forma: identificamos o quadrado de Durfee da partição e o retângulo de altura $n$ e base $k$ ao lado deste quadrado. O fator é identificado como segue: consideremos a altura da primeira pilha (disposta ao lado do retângulo de altura $n$ 
e base $k$ ) e a largura desta pilha. A largura é o expoente de $t$, a altura é a posição do fator e o expoente de $q$ é

$$
2 \times \text { altura } \times \text { largura } .
$$

Repita este procedimento para cada pilha.

Fazendo $q=1 \mathrm{em}$ (1.3), temos que $P_{n}^{k}=F_{n}$ onde $F_{n}$ é o número de Fibonacci de posição $n$. Assim, segue do procedimento acima o seguinte resultado:

Teorema 3.1. O total de partições autoconjugadas Frobenius de alternância ímpar no caso que $k$ é ímpar (par, no caso em $k$ é par) com maior parte menor do que ou igual a $N+k$, onde o elemento do topo $\geq k$ é igual ao número de Fibonacci de posição $N$.

Observamos que as partições Frobenius de alternância ímpar com maior parte igual a $N+1$ são geradas por $f_{16}(q, t)$ e são um caso particular das partições autoconjugadas Frobenius de alternância ímpar, caso $k$ seja ímpar (par), com maior parte $N+k$ e o elemento do topo $\geq k$, quando fazemos $k=1$. O caso $k=0$, do Teorema (3.1) fornece um resultado ja obtido por Santos em [8].

Corolário 3.1.1. O total de partições autoconjugadas Frobenius de alternância par com maior parte menor do que ou igual a $N$ é igual ao número de Fibonacci de posição $N$.

Abstract. In this paper, we give a new combinatorial interpretation for the Fibonacci Numbers in terms of restricted partitions by making use of the Frobenius Symbol. Also we give the proof of an explicit formula for a family of polynomials given by Santos in [9].

\section{Referências}

[1] G.E. Andrews, q-Series: Their development and application in analysis, number theory, combinatorics, physics an computer algebra, "CBMS Regional Conference Series in Math.", American Mathematical Society, Providence Rhode Island, Number 66, pp 87-93, 1986.

[2] G.E. Andrews e R.J. Baxter, Lattice gas generalization of the hard hexagon model. III q-trinomio coeficientes, J. Stat. Phys., 47 (1987) 297-330.

[3] G.E. Andrews. Generalized partitions, Mem. Amer.Math. Soc., 301 (1984) 144.

[4] G.E. Andrews, "The theory of partitions", Encyclopedia of Mathematics and its Applications, Vol.2, Cambridge University Press, London and New York, 1985.

[5] G.E. Andrews, Combinatorics and Ramanujan's "lost" notebook, "London Mathematical Society Lecture Notes Series", Cambridge University Press, London, Number 103, pp 1-23, 1985. 
[6] G.E. Andrews, "Number Theory", Dover Publications, New York, 1994.

[7] P. Mondek, "Identidades de Slater: Novas identidades e interpretações combinatórias," Tese de Doutorado, IMECC-UNICAMP, 1997.

[8] J.P.O. Santos. On the combinatorics of polynomial generalizations of RogersRamanujan type identities, Discrete Mathematics, 254 (2002) 497-511.

[9] J.P.O. Santos, "Computer algebra and identities of the Rogers-Ramanujan type", Ph.D. Thesis, Pennsylvania State University, 1991.

[10] L.J. Slater, Further identities of the Rogers-Ramanujan type, Proc. London Math. Soc. (2), 54 (1952) 147-167. 
